\title{
ALTERNATIVE SOURCE TERMS FOR SWAN IN THE COASTAL REGION
}

\author{
James Salmon, Leo Holthuijsen, Pieter Smit, Gerbrant van Vledder and Marcel Zijlema ${ }^{1}$
}

\begin{abstract}
This paper presents the application of new source terms in SWAN for the dominant water wave physics in the coastal zone: depth-induced breaking and triad wave-wave interactions. We present results demonstrating increased modelling skill in the prediction of bulk wave parameters e.g. significant wave height and of the spectral shape compared to currently used defaults, particularly in cases with horizontal bathymetries. These preliminary results suggest a greater range of applicability of these source terms for operational applications.
\end{abstract}

Keywords: shallow wave breaking; triad wave-wave interactions; spectral wave modelling; SWAN

\section{INTRODUCTION}

SWAN is a third-generation wave model (Booij et al., 1999) specifically developed for operational use in the coastal zone. Although it has been used over the past 15 years with numerous developments including the support for parallelization and unstructured grids, the representation for the dominant shallow water-wave physics: depth-induced wave breaking and triad wave-wave interactions have remained virtually unchanged despite known shortcomings, particularly in the estimation of wave period measures, and alternatives available.

The default source term for depth-induced wave breaking in SWAN is derived from the work of Battjes and Janssen (1978). They use a truncated Rayleigh distribution to represent the random wave heights and couple this to the dissipation of a single breaking wave, considered analogous to the dissipation of a 1D bore. The resulting bulk dissipation is then distributed proportionally to the energy density spectrum as wave-breaking is assumed to not affect the spectral shape (Beji and Battjes, 1993). From the observations of Battjes and Stive (1985), an averaged constant value for the scaling parameter $\gamma_{B J}=0.73$ is used. However, numerous studies have shown the underestimation of the significant wave height of locally generated waves over (near-) horizontal bathymetries with this default parameterization for depth-induced wave breaking e.g. van Vledder et al. (2008). Van der Westhuysen (2010) addresses this issue by noting the equilibrium balance between wave growth, white capping and depth-induced wave breaking under these conditions. In his proposed parameterization, he reduces the dissipation from depth-induced breaking in these conditions by introducing a scaling based on wave non-linearity. Conversely, for non-locally generated waves propagating and breaking over horizontal bathymetries, for example swell over a reef, observations suggest that the default source term should over-estimate the significant wave height. Under such conditions, field observations suggest $\gamma_{B J}=0.55$ (Nelson, 1997). This dichotomy in modelling performance over horizontal bathymetries is discussed in greater detail by Salmon et al. (2014).

Although a strong coupling between depth-induced breaking and nonlinear triad wave-wave interactions has been demonstrated in a number studies (e.g. Chen et al., 1997), much success has been demonstrated by de-coupling these processes. The default source term in SWAN for the triad source term is the Lumped Triad Approximation model (LTA; Eldeberky, 1996). As the bi-spectral evolution is not computed in phase-averaged wave models, the LTA uses a parameterization for the local bispectrum based on the local depth and the spectrum itself. For computational efficiency, the LTA only considers collinear self-self interactions which results in a poor representation of the spectral shape and related integral quantities of e.g. mean wave period. Other studies (e.g. Booij et al., 2009) have also shown the inability of the LTA model to reproduce the transitions to the universal $k^{-4 / 3}$ high frequency tail in shallow water ( $k d=1$ ) which is demonstrated in a number of observations (e.g. Smith, 2004).

In this paper, we address these issues by comparing alternative source terms for the defaults currently used in SWAN. We compare the depth-induced breaking parameterization of Salmon et al. (2014) over observations taken over a 1:30 laboratory slope (Smith, 2004) and laboratory observations of an idealized horizontal reef (Jensen, 2002). We then compare a version of the Stochastic Parametric model based on Boussinesq equations (SPB; Becq-Girard et al., 1999) model for the triad source term over these cases. Finally, we present a comparison between the default shallow water physics and these alternatives over observations taken at Haringvliet (Ris et al., 1999). The intention of this work is to

\footnotetext{
${ }^{1}$ Environmental Fluid Mechanics, Delft University of Technology, Stevinweg 1, Delft, $2628 \mathrm{CN}$, the Netherlands
} 
demonstrate preliminary results of using these alternative source terms and the limitations of the currently used default source terms.

\section{NUMERICAL MODEL}

SWAN (40.91A) solves for the wave action density in five dimensions i.e., $N(\sigma, \theta ; x, y, t)=E(\sigma, \theta ; x, y, t) / \sigma$ (Bretherton and Garrett, 1968) where $E(\sigma, \theta ; x, y, t)$ is the energy density of the random surface elevation as the function of the relative radian frequency, $\sigma$ and the spectral direction, $\theta$ at each spatial location $(x, y)$ as a function of time, $t$. The wave spectrum evolution is given by the Eulerian action balance:

$$
\frac{\partial N}{\partial t}+\frac{\partial c_{g, x} N}{\partial x}+\frac{\partial c_{g, y} N}{\partial y}+\frac{\partial c_{g, \sigma} N}{\partial \sigma}+\frac{\partial c_{g, \theta} N}{\partial \theta}=\frac{S}{\sigma}
$$

The terms on the left-hand side of Eq. (1) represent the conservative wave kinematics with the terms representing, respectively, the local temporal change of the wave action density and the propagation of wave action in four dimensions with propagation velocities $c_{g, x}, c_{g, y}, c_{g, \sigma}$ and $c_{g, \theta}$. The second and third terms of Eq. (1) account for depth- and current-induced shoaling and energy bunching, whereas the fourth and fifth terms represent, respectively, the shifting of $\sigma$ due to variations in depth and current (the Doppler effect) and the propagation of wave action in $\theta$-space due to depth- and current- induced refraction.

The right hand side of Eq. (1) represents the wave dynamics through the summation of a number of source and sink terms which represent the generation of waves due to wind, the redistribution of the wave energy by nonlinear wave-wave interactions (quadruplet and triad interactions) and the dissipation due to white capping, bottom friction and depth-induced breaking.

We use the default settings of the latest public domain version of SWAN (41.01; swan.tudelft.nl) apart from the applied depth-induced breaking and triad interaction source terms; the focus of this paper. Wave generation due to wind is computed with the source term of Komen et al. (1984) with the wind drag coefficient of Zijlema et al. (2012). For the nonlinear quadruplet wave-wave interactions, the Discrete Interactions Approximation (DIA; Hasselmann et al., 1985) as scaled by the WAMDI group (1988) for shallow water is used. For computational efficiency, this source term is switched off for 1D cases as quadruplets do not play a role in uni-directional waves. For the sink terms, white capping is represented by the pulse model of Hasselmann et al. (1973) as modified by the WAMDI group (1988) and weighted to higher frequencies as suggested by Rogers et al. (2003), and for bottom friction, the model of Hasselmann et al. (1973) with a bottom friction coefficient of $0.038 \mathrm{~m}^{2} \mathrm{~s}^{-3}$ (Zijlema et al., 2012) is used. The source terms for depth-induced wave breaking and nonlinear triad wave-wave interactions are described in the following sections.

For the laboratory cases, a frequency resolution of $\Delta f=0.05 f$ and a directional resolution of $\Delta \theta=0.5^{\circ}$ is used. For the field cases, $\Delta f=0.1 f$ (constrained by the DIA) and $\Delta \theta=10^{\circ}$ is used. For terminating the iterative SWAN computations, the curvature-based criterion of Zijlema and van der Westhuysen (2005) are applied.

\section{ALTERNATIVE SOURCE TERMS}

\section{Depth-induced wave breaking}

The default parameterization for depth-induced breaking is the model introduced by Battjes and Janssen (1978) who estimated the bulk dissipation by combining a truncated Rayleigh distribution to represent the random wave heights with the dissipation of a single breaking wave:

$$
\varepsilon_{B J}=-1 / 4 \alpha_{B J} \bar{f} Q_{b} \rho g H_{\max }^{2}
$$

where $\alpha_{B J} \approx 1$ is a calibration coefficient (default $\alpha_{B J}=1$ ), $\bar{f}$ is the mean wave frequency, $\rho$ is the water density, $g$ is the gravitational acceleration and $H_{\max }$ is the maximum wave height. $Q_{b}$ is the fraction of breakers and is dependent on the root-mean-square wave height, $H_{r m s}$ and $H_{\max }$ : 


$$
\frac{1-Q_{b}}{\ln Q_{b}}=-\left(\frac{H_{r m s}}{H_{\max }}\right)^{2}
$$

Beji and Battjes (1993) observed that the shape of the spectrum seemed unaffected by depthinduced breaking and therefore a spectral distribution distributed as proportional to the spectrum is applied (e.g. van Vledder et al., 1994):

$$
S_{b}(\sigma, \theta)=\varepsilon_{B J} E(\sigma, \theta) / E
$$

where $E$ is the total wave energy.

As $\alpha_{B J}=1$ is a commonly applied default, the only remaining calibration parameter is $H_{\max }=\gamma_{B J} d$. Battjes and Janssen (1978) show reasonable results when $\gamma_{B J}=0.80$ was applied, however $\gamma_{B J}=0.73$, an average taken from Battjes and Stive (1985), is the default setting in SWAN. For convenience, we subsequently refer to this as the BJ model.

Many studies have attempted to scale $\gamma$, the ratio of a characteristic wave height to local depth, with a variety of parameters including local bottom slope $(\beta)$, local normalized wave number $(k d)$, local and offshore wave steepness etc. However, recent studies (e.g. Rattanapitikon, 2007; Salmon et al., 2014) demonstrate insignificant improvements by using these alternative expressions for $\gamma$ in comparison to the BJ model. This is typically due to the compensation of improved modelling skill under certain conditions at the expense of model performance over others; particularly over horizontal bathymetries.

Salmon et al. (2014) show promising results by introducing a joint dependency on both local bottom slope and local normalized wave number for $\gamma_{B J}$ :

$$
\gamma_{\beta-k d}=\gamma_{\beta} / \tanh \left(\gamma_{\beta} / \gamma_{k d}\right)
$$

where $\gamma_{\beta}=0.54+7.59|\nabla d| \geq 0$ represents the $\beta$-dependency on the absolute value of the local bottom slope (determined from the computational grid) and $\gamma_{k d}=-8.06+8.09 \tilde{k} d \geq 0$ represents the $\tilde{k} d$ - dependency where $\tilde{k}=k_{-1 / 2}$ is the characteristic wave number determined from the local spectrum (WAMDI group, 1988). In their scaling, they argue that in shallow water, wave behaviour converges to that of a solitary wave and therefore the scaling with $\tilde{k} d$ becomes irrelevant. Over horizontal bottoms, numerous theoretical limits for the crest height of solitary waves have been estimated (e.g. McCowen, 1894; Longuet-Higgins, 1974) that suggest over an average depth, $\bar{d}, 0.56 \leq H / \bar{d} \leq 0.60$. These limits are similar to the lower limit of $\gamma_{\beta-k d, l o w}=0.54$. At larger values of $\tilde{k} d$ (typically $\tilde{k} d>\sim 1$ ), typical of intermediate to deep water, waves no longer behave as solitary waves and become dependent on both the local bottom slope and the local normalized characteristic wave number. In this range of $\tilde{k} d$ values, typically $\gamma_{\beta-k d}>0.73$. This reduces dissipation due to depth-induced breaking as demonstrated by van der Westhuysen (2010).

To account for 'real' wave conditions which are not typically 1D and long-crested (directionally narrow), even in the case of swell, Salmon et al. (2014) introduced a modification to Eq. 2 and 3 to include wave directionality:

$$
\varepsilon_{B J}=-1 / 4 K_{\theta} \alpha_{B J} \bar{f} Q_{b} \rho g H_{\max }^{2}
$$

with

$$
\frac{1-Q_{b}}{\ln Q_{b}}=-\left(\frac{H_{r m s} / \sqrt{K_{\theta}}}{H_{\max }}\right)^{2}
$$

where $K_{\theta}=\sigma_{\theta} / \sigma_{\theta}^{*} \geq 1$ represents the number of directional partitions which are each assumed to be sufficiently long crested so that the $1 \mathrm{D}$ bore assumption can be applied, $\sigma_{\theta}$ is the directional width of 
the spectrum (Kuik et al., 1988) and $\sigma_{\theta}^{*}$ is the limiting value at which the $1 \mathrm{D}$ bore assumption holds. From the field observations used by Salmon et al. (2014), optimal results were shown with $\sigma_{\theta}^{*}=15^{\circ}$. The joint parameterization combined with the directional partitioning is subsequently referred to as the $\beta-k d$ model. Note that this is not the same as the spectral partitioning used to differentiate between different wave systems.

\section{Nonlinear triad wave-wave interactions}

The default parameterization for the nonlinear triad wave-wave interaction is the Lumped Triad Approximation (LTA; Eldeberky, 1996; his Eq. 7.25; Booij et al., 1999; their Eqs, A6-A8) which is based on the extended Boussinesq model of Madsen and Sørensen (1993). The source term can be expressed as:

$$
S_{L T A}\left(\sigma_{1}\right)=S_{L T A}^{+}\left(\sigma_{1}\right)-2 S_{L T A}^{+}\left(2 \sigma_{1}\right)
$$

with

$$
S_{L T A}^{+}\left(\sigma_{1}, \theta\right)=\max \left\{0,2 \pi \alpha_{L T A} C_{1} C_{g, 1} J_{\sigma_{1} / 2, \sigma_{1} / 2}^{2}|\sin \varphi| \times\left[E^{2}\left(\frac{\sigma_{1}}{2}, \theta\right)-2 E\left(\frac{\sigma_{1}}{2}, \theta\right) E\left(\sigma_{1}, \theta\right)\right]\right\}(9)
$$

where $\alpha_{L T A}$ is a calibration coefficient (default $\alpha_{L T A}=0.05$ ), $c_{1}$ and $c_{g, 1}$ are the phase velocity and group velocity of the frequency component $\sigma_{1}$ and $\varphi=\frac{\pi}{2}\left\{\tanh \left(\frac{0.2}{U r}\right)-1\right\}$ is the parameterization for the bi-phase of the self-self interactions of $\sigma_{1}$ as a function of the Ursell number, Ur (Eldeberky, 1996; Doering and Bowen, 1995). The interaction coefficient is taken from Madsen and Sørensen (1993):

$$
J_{\sigma_{1} / 2, \sigma_{1} / 2}=\frac{R_{\sigma_{1} / 2, \sigma_{1} / 2}}{S_{\sigma_{1}}}=\frac{\left(k_{\sigma_{1} / 2}+k_{\sigma_{1} / 2}\right)^{2}\left[\frac{1}{2}+\left(g d k_{\sigma_{1} / 2} k_{\sigma_{1} / 2}\right)^{-1} \sigma_{\sigma_{1} / 2} \sigma_{\sigma_{1} / 2}\right]}{-2 k_{\sigma_{1}} g^{-1}\left[g d+\frac{2}{15} g d^{3} k_{\sigma_{1}}^{2}-\frac{2}{5} d^{2} \sigma_{1}^{2}\right]}
$$

An alternative parameterization, the Stochastic Parametric model based on Boussinesq equations (SPB) model by Becq-Girard et al. (1999) relaxes the constraint of only considering the self-self interactions and considers all interacting frequency components:

$$
S_{S P B}\left(\sigma_{1}\right)=8 \pi c_{g, 1} S_{\sigma_{1}}^{-1}\left[\int_{0}^{\sigma_{1}} R_{\sigma_{2}, \sigma_{1-2}} B_{2,1-2}^{i m} d \sigma_{2}-2 \int_{0}^{\infty} R_{-\sigma_{2}, \sigma_{1+2}} B_{-2,1+2}^{i m^{*}} d \sigma_{2}\right]
$$

where

$$
B_{2,1-2}^{i m}=\frac{1}{2}\left[J_{2,1-2} E_{2} E_{1-2}-J_{1,-2} E_{1} E_{2}-J_{1,2-1} E_{1} E_{1-2}\right] \mu_{1,2,1-2}(K)
$$

represents the imaginary part of the bispectrum and the indices are introduced for brevity i.e. $E_{1-2}=E\left(\sigma_{1}-\sigma_{2}\right)$. The parameter $\mu_{1,2,1-2}(K)=K\left[(\Delta k)^{2}+K^{2}\right]^{-1}$ originates from the statistical closure hypothesis of Holloway (1980) and represents a broadening of the resonance conditions where $\Delta k$ is the wavenumber mismatch and $K$ is a calibration parameter. Becq-Girard et al. (1999) suggested a dimensional scaling of $K=0.95 k_{p, 0}-0.75$ where $k_{p, 0}$ is the peak offshore wave number. The interaction coefficients $J$ are computed from Eq. (10), however are no longer restricted to the self-self interactions as in the LTA model, i.e., $J_{2,1-2} \equiv J_{\sigma_{2}, \sigma_{1}-\sigma_{2}}$.

In order to implement the SPB model in SWAN, we made three modification to the SPB model presented above. As $K$ is expressed as a function of an offshore parameter, which is not well-defined for 2D applications, we scale $K$ as a function of the equivalent local parameter i.e., local peak wave number $K_{m S P B}=0.95 k_{p}-0.75$. Furthermore, to distribute the 1D directionally integrated source term over the directional bins i.e., $S_{S P B^{*}}(\sigma, \theta)$, we assume a collinear approximation where the dominant contributions are assumed to only occur over a narrow finite directional width centred about the spectral directional bin. To allow for these two modifications, we also introduced an additional calibration 
parameter which acts over the complete source term i.e., $S_{m S P B}=\alpha_{m S P B} S_{S P B^{*}}$. From trial computations over a number of laboratory cases, a value of $\alpha_{m S P B}=0.75$ is used in this study. We refer to this model as the mSPB (modified SPB) model.

\section{OBSERVATIONS}

To verify the performance of the proposed alternative source terms, we compared the model performance of the default source terms and the alternative source terms to two laboratory cases and one field case as described in the following.

\section{Laboratory cases}

\section{Smith (2004)}

The Smith (2004) laboratory data set consists of 31 cases performed at the US Army Engineer Research and Development Centre, Coastal and Hydraulics Laboratory encompassing a variety of spectra including unimodal and bimodal incident spectra with varying degrees of wave steepness and spectral width. The wave flume consisted of a 1:30 slope and waves were measured at 10 locations, nine of which are located on the slope (Fig. 1A).

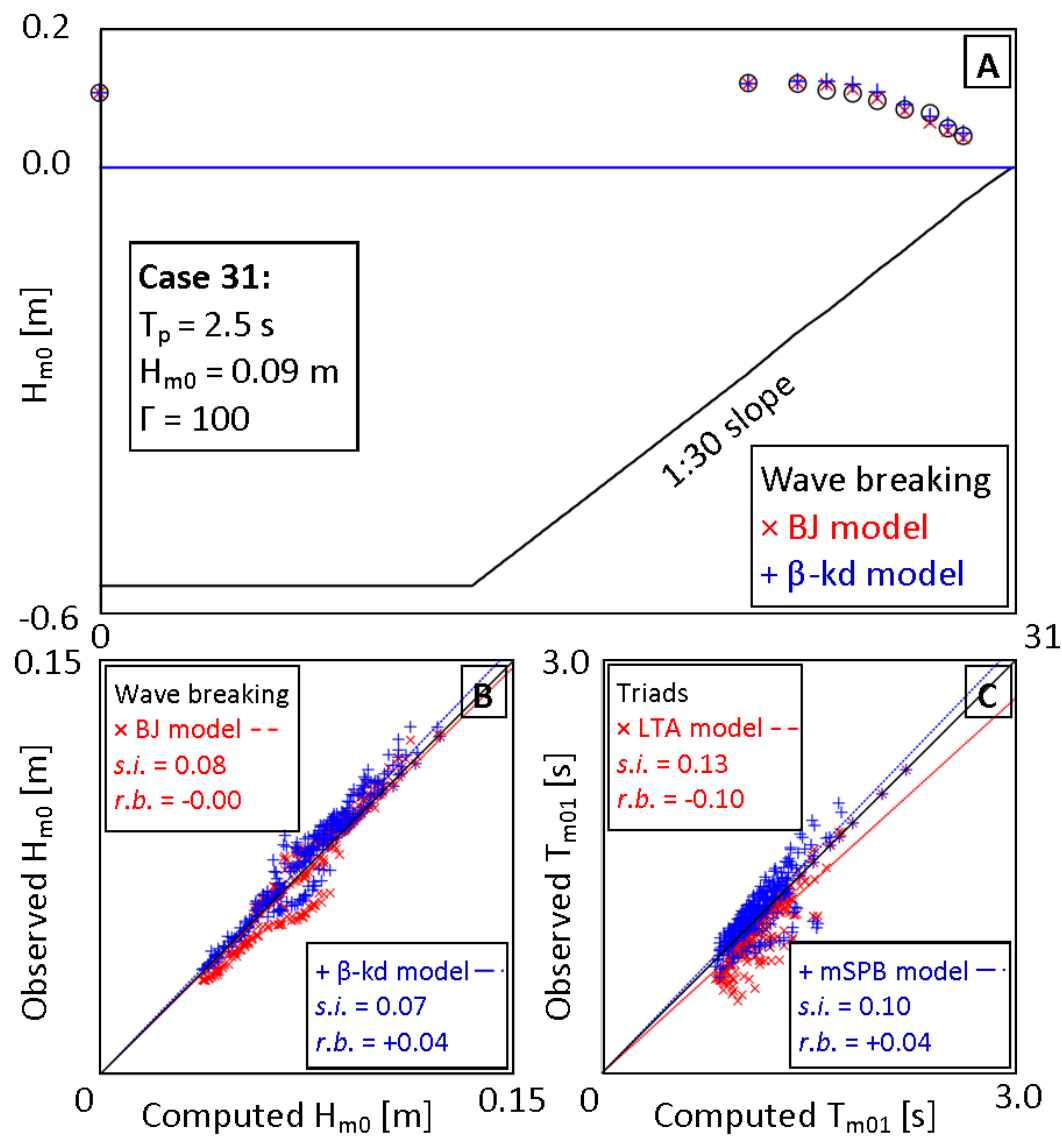

Figure 1. Experimental set-up and computational results for the Smith (2004) data set. Top panel (A) shows the flume profile and measuring locations with computed $H_{m 0}$ using the $\mathbf{B J}$ (red $\times$ ) and $\beta-k d$ (blue +) breaker models for Case 31 with the LTA triad model. Panel B shows the performance of these breaker models in predicting $H_{m 0}$ for all 31 cases. Panel $\mathbf{C}$ shows the performance in predicting $T_{m 01}$ of the LTA triad model (red $x$ ) and the mSPB triad model (blue + ) with the BJ breaker model. The performance metrics: s.i. and r.b. are presented in Panels B and C as defined in Eqs. (13) and (14).

Irregular waves are generated at a piston wave generator as either unimodal or bimodal spectra described with the parametric TMA spectral shape (Bouws et al., 1985). For the case of the doublepeaked spectra, the spectra is formed by the linear combination of the two underlying TMA spectra. For 
these cases, two-thirds of the total energy is contained at the higher peak, set at a $1 \mathrm{~Hz}$. For all cases, the peak wave period varied between $1.0 \leq T_{p} \leq 2.5 \mathrm{~s}$ with a significant wave height of either $H_{m 0}=0.06$ or $0.09 \mathrm{~m}$. The spectral peakedness parameter, $\Gamma$ varied between three values from 3.3 for a broad spectrum, 20 and 100 for a narrow spectrum.

\section{Jensen (2002)}

The Jensen (2002) laboratory data set consists of a total of 301 cases with regular waves and 110 tests with irregular waves over 4 different reef approach slopes (varying between 1:0.5, 1:1, 1:1 ' $\mathrm{s}$ 'shape and 1:2) and a horizontal section. Waves were measured at 14 different locations, however wave measurements at only four locations over the horizontal are considered here (Fig. 2A).

We chose a random selection of 25 cases from the 110 irregular wave cases. The corresponding range of incident peak frequencies for the unimodal JONSWAP spectra were $1.4 \leq T_{p} \leq 2.6 \mathrm{~s}$ with a significant wave height between $0.11 \leq H_{m 0} \leq 0.21 \mathrm{~m}$.
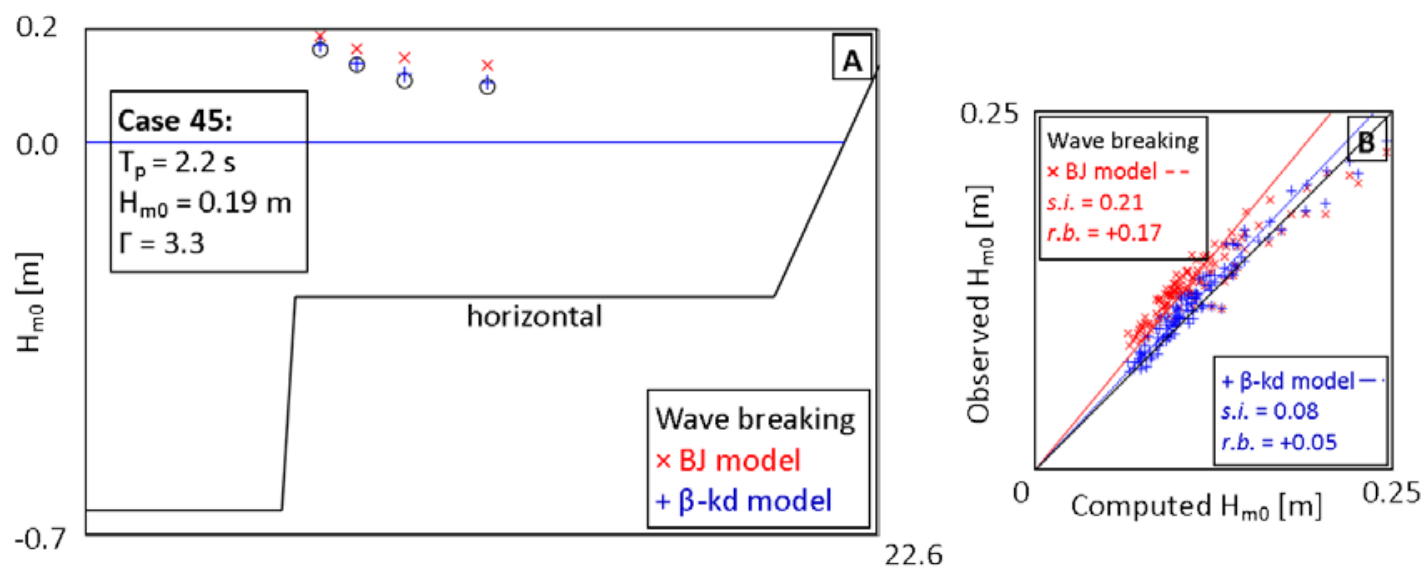

Figure 2. Experimental set-up and computational results for the Jensen (2002) data set. Panel A shows the flume profile and measuring locations with computed $H_{m 0}$ using the $\mathbf{B J}$ (red $\mathbf{x}$ ) and $\beta-k d$ (blue +) breaker models for Case 45 with the LTA triad model. Panel B shows the performance of these breaker models in predicting $H_{m 0}$ for all 25 cases (random selection). Wave period measures were not available for this data set. The performance metrics: s.i. and r.b. are presented in Panel B as defined in Eqs. (13) and (14).

\section{Field cases}

\section{Haringvliet (October 14, 1982)}

The Haringvliet is characterized as a relatively shallow $10 \times 10 \mathrm{~km}^{2}$ bay in the southwest of the Netherlands. It is partially protected by a shoal (the 'Hinderplaat') which extends across half of its entrance (Fig. 3). Four cases at 21:00, 22:00, 23:00 and 00:00 UTC are selected from observations taken from a north-westerly local storm over the southern North Sea on October 14, 1982. These cases were chosen as negligible currents $(<0.25 \mathrm{~m} / \mathrm{s})$ occurred during the observations, the wind conditions were fairly constant, the waves were relatively high and the water level was sufficiently low to observe the generation of a secondary peak.

Over the observation period, the minimum water depth over the shoal varied between 1.0 and $2.2 \mathrm{~m}$ and the incident significant wave height varied between $3.2 \leq H_{m 0} \leq 3.6 \mathrm{~m}$. The wind speed varied between $14.0<U_{10}<17.0 \mathrm{~m} / \mathrm{s}$ from $\sim 300^{\circ} \mathrm{N}$. Further details are given in Ris et al. (1999). 

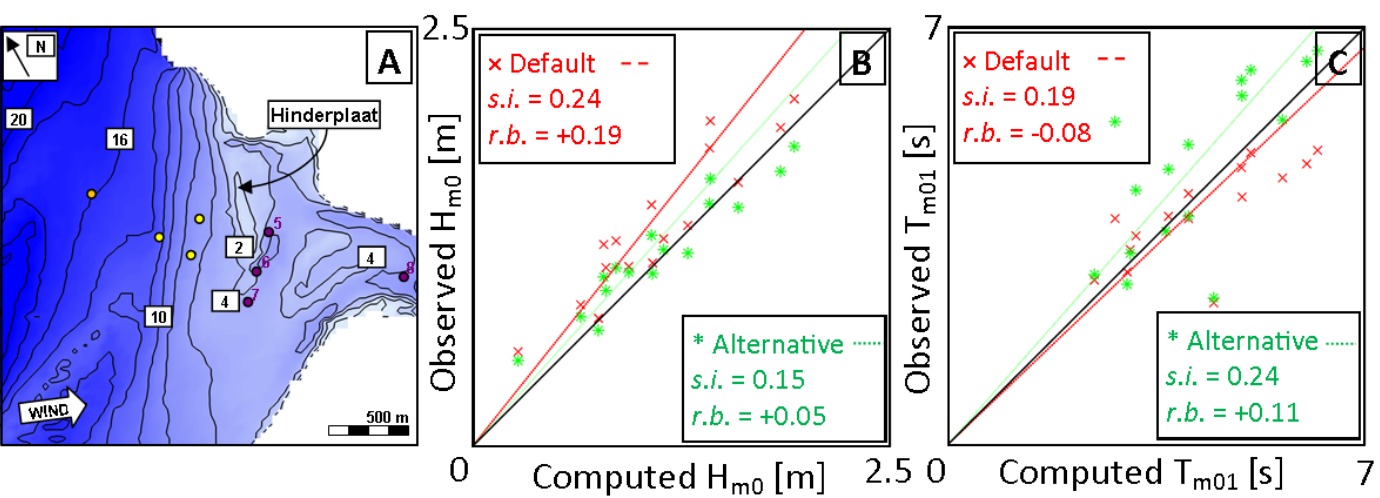

Figure 3. Bathymetry and computational results for the Haringvliet (October 14, 1982) data set. Panel A provides the bathymetry for the region at $+1 \mathrm{~m}$ above Amsterdam Ordnance Datum (NAP) with contour lines given at $2 \mathrm{~m}$ intervals. Wave observation stations are shown as dots. Only locations shown in purple $(5,6,7,8)$ are considered. Panel B shows the performance of default SWAN (BJ + LTA; red $x$ ) and the alternative source terms $\left(\beta-k d+\mathrm{mSPB}\right.$; green *) in predicting $H_{m 0}$. Panel $\mathrm{C}$ shows the equivalent for $T_{m 01}$. The performance metrics: s.i. and r.b. are presented in Panels B and C as defined in Eqs. (13) and (14).

\section{RESULTS}

\section{Depth-induced wave breaking}

The default source terms for depth-induced breaking and triad non-linear interactions were applied to the above cases. The results over the sloping Smith (2004) data set is shown in Fig. 1 (in blue). The comparison between the observed and the predicted significant wave height shown in the scatter plot in Fig. 1B shows good modelling skill over relatively steep slopes as demonstrated in previous studies (e.g. Rattanapitikon, 2007). For illustration purposes, Case $31\left(H_{m 0}=0.09 \mathrm{~m}, T_{p}=2.5 \mathrm{~s}, \Gamma=100\right)$, shown in Fig. 1A, illustrates the typically good agreement between the observations and model results for $H_{m 0}$. The modelling skill for each test case can be expressed by calculating performance metrics: scatter index ( s.i. ) and the relative bias ( r.b. ) as used in previous studies (e.g. Janssen et al., 1984):

$$
\text { s.i. }=\sqrt{N \sum_{i=1}^{N}\left(\chi_{\text {comp }}^{i}-\chi_{o b s}^{i}\right)^{2}} / \sum_{i=1}^{N}\left(\chi_{o b s}^{i}\right)
$$

and

$$
r . b .=\sum_{i=1}^{N}\left(\chi_{\text {comp }}^{i}-\chi_{o b s}^{i}\right) / \sum_{i=1}^{N}\left(\chi_{o b s}^{i}\right)
$$

where comp and obs refer to the predicted and observed value of the quantity $\chi$ over sample size $N$. In our study, $\chi$ relates to the significant wave height, $H_{m 0}$ :

$$
H_{m 0}=4 \sqrt{m_{0}}=4\left[\int E(\sigma) d \sigma\right]^{1 / 2}
$$

or the mean wave period $T_{m 01}$ :

$$
T_{m 01}=m_{0} m_{1}^{-1}=2 \pi \int E(\sigma) d \sigma / \int \sigma E(\sigma) d \sigma
$$

For each data set, the performance metrics were computed for each case, and then averaged over the number of cases to give an averaged s.i. and averaged r.b. . The corresponding values over the Smith (2004) data set with default source terms are s.i. $=0.08$ and $r . b .=-0.00$.

Conversely, over the horizontal bathymetries of the Jensen (2002) data set, as shown in Fig. 2B, a large over-estimation of the significant wave height is shown. The corresponding performance metric were s.i. $=0.21$ and r.b. $=+0.17$. This overestimation $H_{m 0}$ of can be seen in the example shown in Fig. 2A. In comparison with the sloping laboratory cases, the magnitude of the total error is more than double and the relative bias forms a significant contribution to the total error. 
Also shown in Figs. 1B and 2B is the performance of the $\beta-k d$ model with the LTA source term (in green). Over the Smith (2004) data set, the performance is comparable to the BJ model with s.i. $=0.07$ and r.b. $=+0.04$ whereas over the Jensen (2002) data set, the $\beta-k d$ model is also shown to provide comparable modelling performance as shown in the Smith (2004) data set with s.i. $=0.08$ and r.b. $=+0.05$.

\section{Nonlinear triad wave-wave interaction}

Although the previous comparison illustrates reasonable results for the prediction of $H_{m 0}$ over relatively steep slopes (1:30) with the default source terms, for the prediction of integral parameters which are more sensitive to the spectral shape e.g. mean wave period measures, the performance is relatively poorer. This is due to the poor representation, if at all, of the nonlinear triad interactions. This is illustrated in Fig. 1C for the LTA model with s.i. $=0.13$, of which a significant amount is negative bias $(r . b .=-0.10)$ for the mean wave period $T_{m 01}$. Conversely, the mSPB model appears to give better results with slightly smaller errors $(s . i .=0.10)$ and a significantly reduced bias $(r . b .=+0.04)$.

These differences are illustrated in Fig. 4 where the observed and computed spectra for Case 31 are presented. Following Booij et al. (2009), we scale the energy density so that the universal $k^{-4 / 3}$ tail appears as a horizontal line. It is clear that the LTA is only able to transfer energy to $f_{2^{n} \times p}$ where $n$ is a positive integer. Furthermore, it is clear that for this case these peaks are severely overestimated with energy not removed deeper in the surf zone e.g. Stations 5 and 7. In contrast, the mSPB model provides better agreement with the observations. All higher harmonics are predicted relatively well, including locations deep in the surf zone. In addition, the universal tail is also in better agreement with the observations compared to the LTA model results.
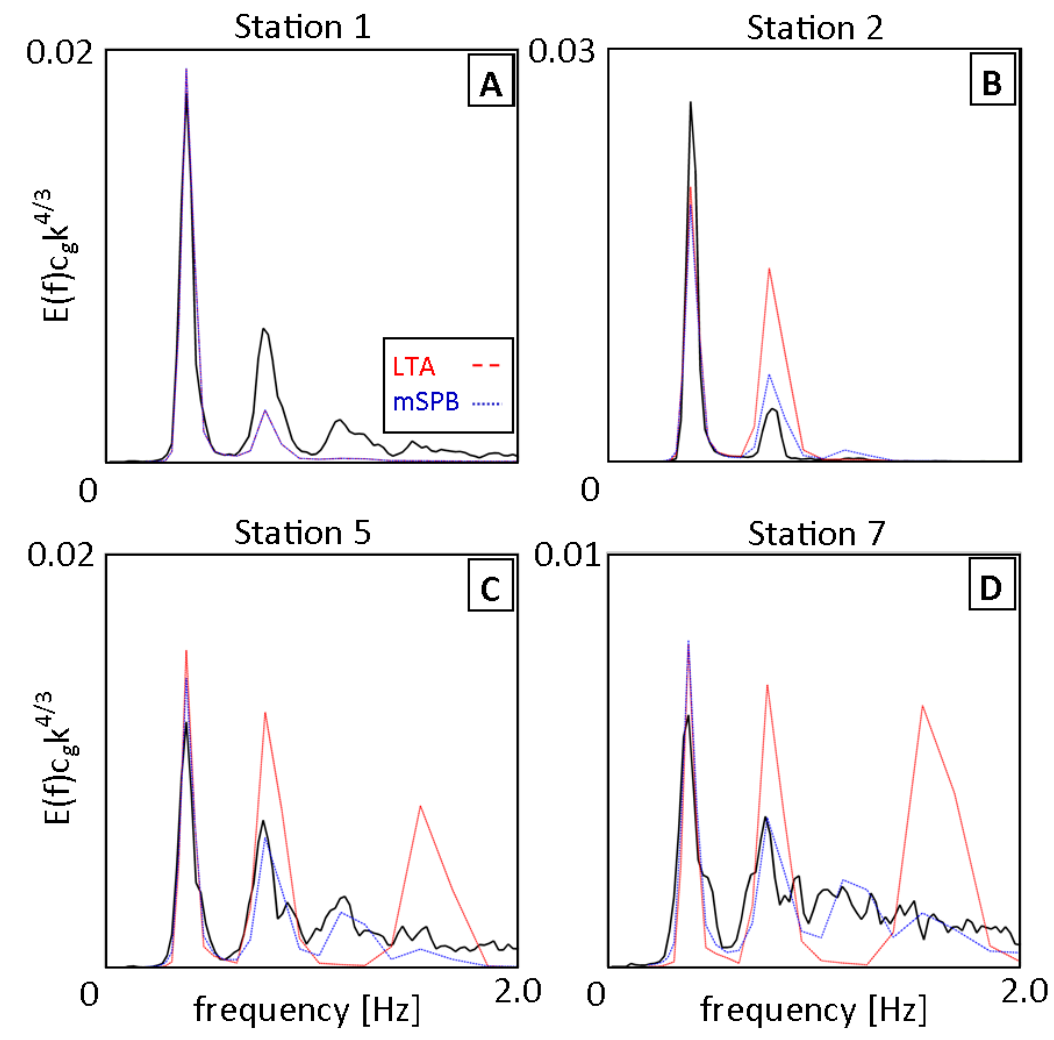

Figure 4. Observed and computed scaled variance density spectra for Case 31 of the Smith (2004) data set. Panels $A$ to $D$ show the evolution of the spectra in the surf zone from at the wave-maker (Location 1) to deep in the surf zone (Location 7). Spectra computed with the LTA are shown in red (--) and the spectra computed with the mSPB are shown in blue (..). Note that the spectra are scaled so that a $k^{-4 / 3}$ tail appears horizontal. 


\section{Combined source terms over a field case}

To verify the combined use of both the $\beta-k d$ and mSPB model, we compared the combined used of these source terms to default SWAN i.e., the BJ and LTA model over the Haringvliet data set. This case represents depth-induced wave breaking over a relatively horizontal shoal (the 'Hinderplaat') as well as the generation of a significant secondary peak behind the shoal (Ris et al, 1999). To reduce the bias from observations taken in deeper water, which would be largely be unaffected by wave breaking and triad interactions, we only considered wave observations taken in depths of $d<6 \mathrm{~m}$ (Locations 5, 6, 7 and 8; shown as purple dots in Fig. 3A).

Fig. 3B demonstrates the model comparison for the prediction of $H_{m 0}$ over the Haringvliet data set. Similar to the Jensen (2002) cases shown in Fig. 2B, a significant over-estimation of $H_{m 0}$ is shown with the default source terms (red; s.i. $=0.24$ and $r . b .=+0.19$ ). These errors are significantly reduced, particularly the relative bias, with the use of the alternative source terms (green; s.i. $=0.15$ and r.b. $=+0.05$ ).

However, compared to the improvements shown in Fig. 1C and Fig. 4, the model comparison shown for $T_{m 01}$ in Fig. 3C demonstrate a decrease in modelling skill with the application of the alternative source term compared to the defaults. Whereas the performance metrics for the default source terms are s.i. $=0.19$ and $r . b .=-0.08$, the equivalent values for the alternative source terms are s.i. $=0.24$ and r.b. $=+0.11$. To further investigate this lack of improvement, the observed and predicted spectra for measurements at 23:00 UTC are shown in Fig. 5.
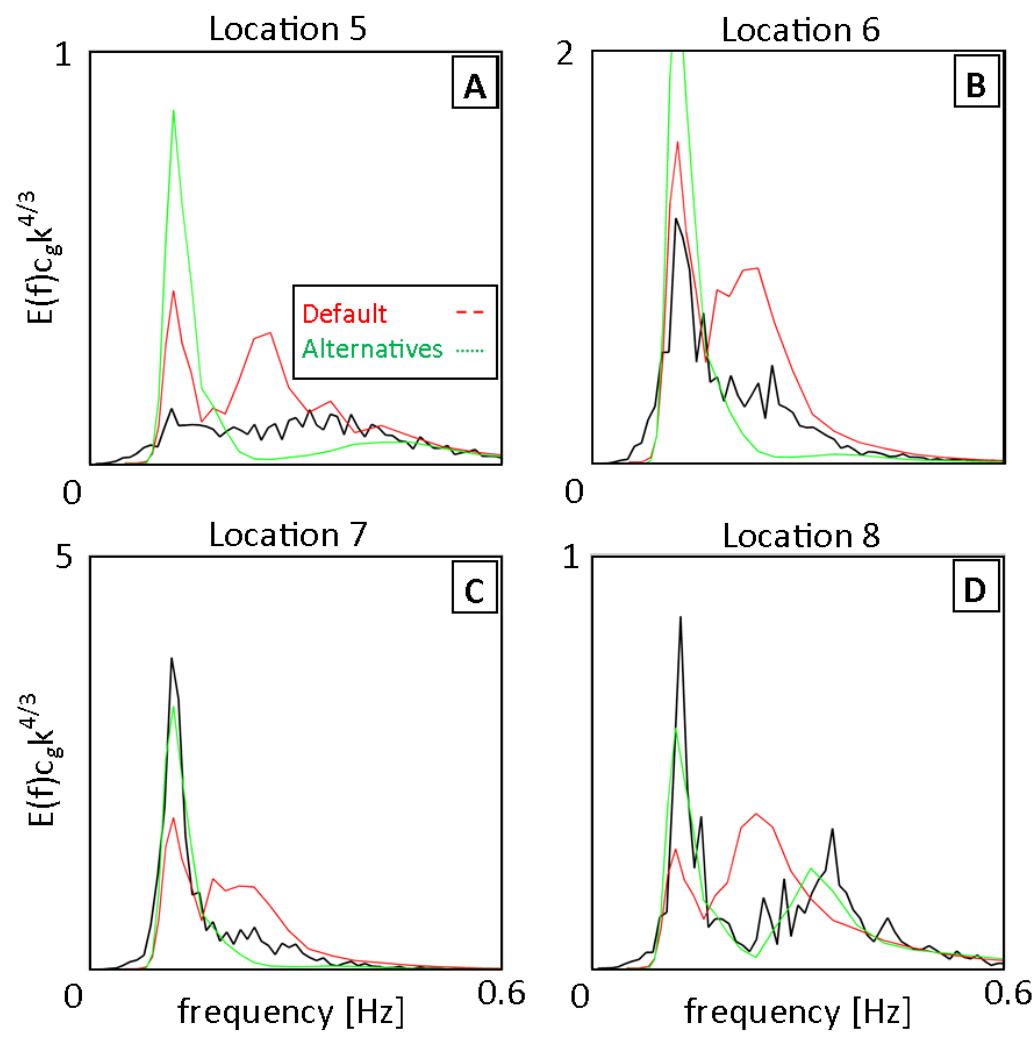

Figure 5. Observed and computed scaled variance density spectra for case 23:00 UTC of the Haringvliet data set at the four most shoreward locations. Spectra computed with the default source terms (LTA + BJ model) are shown in red (--) and the spectra computed with the alternative source terms $(\beta-k d+\mathrm{mSPB})$ are shown in green (..). Note that the spectra are scaled so that a $k^{-4 / 3}$ tail appears horizontal.

At the wave observation locations just behind the shoal i.e., Location 5 and 6, both the LTA and SPB model perform poorly. Whereas the LTA model transfer too much energy to the second harmonic as shown previously in the Smith (2004) data set, the mSPB transfers too little energy to the higher 
harmonics. This results in an underestimation of $T_{m 01}$ with the LTA and an overestimation with the mSPB.

At Location 7, the mSPB model captures the energy at the primary peak well however as seen at Location 5 and 6 the energy at the higher frequencies is also underestimated. However, compared to the LTA which transfers too much energy from the primary peak to the secondary peak, the mSPB provides better agreement with the observed spectral shape.

At a greater distance from the shoal, at Location 8, good agreement between the observed spectra and the mSPB model results is shown. Although slightly underestimated, the primary peak is reproduced relatively well and the amount of energy at the second harmonic is also in good agreement with the observations. Furthermore, the energy at the higher frequencies is also in good agreement with the observations. Conversely, as shown at Location 7, the LTA still transfers too much energy from the primary peak to the second harmonic.

\section{Computational efficiency}

The averaged relative contributions of the depth-induced breaking source term and triad source term over all four cases of the Haringvliet data set is shown in Table 1. It is clear that the default BJ and LTA source terms contribute very little to the total computational cost $(<2 \%$ and $<5 \%$ of the total respectively). The proposed $\beta-k d$ is also shown to be comparable to the $\mathrm{BJ}$ model in terms of computational cost in the $\beta-k d+\mathrm{mSPB}$ column.

\begin{tabular}{|c|c|c|c|c|}
\hline & Default & $B J+m S P B$ & $\beta-k d+\mathrm{mSPB}$ & $\mathrm{BJ}+\mathrm{DCTA}^{1}$ \\
\hline $\begin{array}{l}\text { Total } \\
\text { Wave breaking } \\
\text { Triad interactions } \\
\text { Other }\end{array}$ & $\begin{array}{c}100.00 \\
1.45 \\
4.58 \\
93.97 \\
\end{array}$ & $\begin{array}{c}4310.92 \\
1.56 \\
4197.82 \\
111.54 \\
\end{array}$ & $\begin{array}{c}4415.90 \\
1.53 \\
4304.00 \\
110.37\end{array}$ & $\begin{array}{c}462.23 \\
1.46 \\
362.01 \\
98.76 \\
\end{array}$ \\
\hline Av. \# Iterations & 8.50 & 9.35 & 9.35 & 9.00 \\
\hline
\end{tabular}

However, much of the increased computational cost of using alternative source terms, as shown in the last three columns is due to the use of alternative triad source terms to the LTA model. In the case of the mSPB model, the source term is up to three orders of magnitude more expensive. Such a steep increase in computational cost is not surprising due to the introduction of the integral over all other frequencies represented by Eq. 11 and a more complex representation of the bi-spectrum as given in Eq. 12. This increase in computational cost results in computations up to 45 times more expensive for a complete model run. In the final row, the average number of iterations for convergence over the four Haringvliet cases is given. A slight change to the convergence behaviour between the LTA and mSPB model is demonstrated. However it represents, at most, an additional iteration step with the mSPB model for the Haringvliet cases considered. Therefore, the increased computational cost of the mSPB model can be attributed to the computation of the triad source term rather than a poor convergence behaviour.

\section{DISCUSSION}

The alternative source terms presented here show good agreement with observations made in laboratory conditions. Over the sloping cases of the Smith (2004) data set, the $\beta-k d$ model perform comparably with the BJ model, whereas over the horizontal cases of the Jensen (2002) data set, the over-estimation of the $\mathrm{BJ}$ model is largely reduced with the use of the $\beta-k d$ model resulting in better model performance. Much of this improvement comes from the lower value of $\gamma_{\beta-k d}=\gamma_{0}=0.54$ under the wave conditions represented by the Jensen (2002) data set i.e., a horizontal bathymetry $(\beta=0)$ with non-locally generated waves $(\tilde{k} d<1$ ). These improvements are expected to be applicable to other similar wave environments e.g. the dissipation of swell over horizontal bathymetries such as reefs and intertidal flats. Similar conditions are found in the Haringvliet data set where waves from a local storm 
propagate and break over a fairly flat shoal. For these cases, the $\beta-k d$ model also shows better modelling skill and a reduced bias compared to the BJ model.

Better agreement with the observed spectral shape of the Smith (2004) data set is demonstrated with the mSPB model in comparison to the LTA model. In these cases, the LTA model demonstrates an excessive transfer of wave energy to the higher frequencies which results in an underestimation of the mean wave period. Conversely, the mSPB model does not transfer too much energy to the higher frequencies and reproduces the primary peak well. For the Haringvliet data set, these improvements are only demonstrated at some distance from the Haringvliet shoal (Locations 7 and 8). At locations closer to the shoal, the mSPB model underestimates the wave energy transfer to the higher frequencies whereas the LTA over-predicts this. However, the almost consistent excessive transfer of wave energy to the second harmonic by the LTA model is not reproduced by the mSPB model in agreement with the observations.

The poorer prediction of $T_{m 01}$ by the mSPB model compared to the LTA model over the Haringvliet data set may be due to a number of reasons. In particular, the ad-hoc modification of the SPB model to use $K_{m S P B}\left(k_{p}\right)$ is not physically justified and is dimensional. Therefore it may not be appropriate for scaling the (m)SPB model. Furthermore, the use of $\alpha_{m S P B}$ is determined from simulations over laboratory cases which may not be representative for field cases. Nonetheless, promising results are shown by the mSPB model, particularly in respect to the almost 'out of the box' configuration of the SPB model presented here. In particular, smaller errors in the prediction of higherorder measures for mean wave period are expected with the mSPB model as typically the energy levels at the higher frequencies are better reproduced.

However, a limitation of the application of models such as the mSPB is the increased computational cost. This arises from computing over all interacting frequencies. Although this may result in more physically realistic spectra (e.g. Fig. 4 and Fig. 5), it comes at a computational cost prohibitive for operational use. As an alternative, the computational cost of the DCTA (Booij et al., 2009) is presented in the final column of Table 1. This source term computes a similar integrals as represented by Eq. 11 but is formulated and implemented so that the sum and difference contributions per frequency are computed simultaneously. The difference in computation time between the mSPB and DCTA source terms shows a computational cost of up to an order of magnitude smaller. Therefore, formulation the mSPB model in a similar manner may lead to a similar increase in computational efficiency.

Further gains may be possible by restricting the range of interacting frequency components considered i.e., to a range between the extremes of only considering the self-self interactions, as done in the LTA model, and all interactions, as considered by the mSPB model. This modifications may allow for an efficient and physically more realistic nonlinear triad interaction source term to become available for operational purposes.

\section{CONCLUSIONS}

Alternative source terms for depth-induced wave breaking and nonlinear triad wave-wave interactions have been presented and some preliminary results shown. In general, the $\beta-k d$ model is shown to provide comparable or improved modelling performance for $H_{m 0}$ when compared to the BJ model over the data sets presented. These improvements come at no decrease in modelling performance (over the cases considered) or increased computational cost.

The mSPB model is shown to provide better agreement with the observed spectral shapes from the Smith (2004) data set as well in the Haringvliet data set at locations relatively far from the Hinderplaat shoal when compared to the LTA model, particularly in the prediction of the higher harmonics. In the remaining locations, where the mSPB model performed worse, the mSPB model typically underpredicted the transfer of wave energy to higher frequencies in contrast to the LTA model which overpredicted these transfers. This decrease in modelling performance of the mSPB model is likely due to the ad-hoc use of $K_{m S P B}\left(k_{p}\right)$ and $\alpha_{m S P B}$ which should be re-assessed in future work. Furthermore, the mSPB comes at a computational cost prohibitive for most operational purposes. In future work, this increased computational cost can likely be reduced by implementing the model to compute the sum and difference terms simultaneously and reviewing the interaction frequencies considered. 


\section{ACKNOWLEDGMENTS}

We greatly appreciate receiving the details of the laboratory observations of Jane Smith of the Coastal and Hydraulics Laboratory, US Army Engineer Research and Development Center in Vicksburg (USA). The first author (J.S.) is financially supported by the US Office of Naval Research under Grant N00014-10-1-0453 and Grant N00014-12-1-0534.

\section{REFERENCES}

Battjes, J.A., and J.P.F.M. Janssen. 1978. Energy loss and set-up due to breaking of random waves, Proc. of $16^{\text {th }}$ Int. Conf. on Coastal Engng., ASCE, 569-587.

Battjes, J.A., and M.J.F. Stive. 1985. Calibration and verification of a dissipation model for random breaking waves, J. Geophys. Res., 90, C5, 9159-9167.

Becq-Girard, F., P. Forget, and M. Benoit. 1999. Non-linear propagation of unidirectional wave fields over varying topography, Coast. Eng., 38, 91-113.

Beji, S., and J.A. Battjes. 1993. Experimental investigation of wave propagation over a bar, Coast. Eng., 19, 151-162.

Booij, N., L.H. Holthuijsen, and M.P. Bénit. 2009. A distributed collinear triad approximation in SWAN, Coastal Dynamics 2009, 1-10.

Bouws, E., H. Günther, W. Rosenthal, and C.L. Vincent. 1985. Similarity of the wind wave spectrum in finite depth water 1. Spectral form, J. Geophys. Res., 90, C1, 975-986.

Bretherton, F.P., and C.J.R., Garrett. 1968. Wavetrains in inhomogeneous moving media, Proc. R. Soc. London A, 302(1471), 529-554, doi:10.1098/rspa.1968.0034.

Chen, Y., R.T. Guza, and S. Elgar. 1997. Modeling spectra of breaking surface waves in shallow water, J. Geophys. Res., 102, C11, 25035-25046.

Doering, J.R.C., and A.J. Bowen. 1995. Parameterization of orbital velocity asymmetries of shoaling and breaking waves using bispectral analysis, Coast. Eng., 26, 15-33

Eldeberky, Y. 1996. Nonlinear transformation of wave spectra in the nearshore zone, Ph.D. thesis, Delft University of Technology, Department of Civil Engineering, The Netherlands, 203 pp. also as Commun. Hydr. Geotech. Eng., 96 - 4, 203 pp.

Hasselmann, K., T.P. Barnett, E. Bouws, H. Carlson, D.E. Cartwright, K. Enke, J.A. Ewing, H. Gienapp, D.E. Hasselmann, P. Kruseman, A. Meerburg, P. Müller, D.J. Olbers, K. Richter, W. Sell, and H. Walden. 1973. Measurements of wind-wave growth and swell decay during the Joint North Sea Wave Project (JONSWAP), Dtsch. Hydrogr. Z., Suppl., A8, 12, 95 p.

Hasselmann, S., K. Hasselmann, J.H. Allender, and T.P. Barnett. 1985. Computations and parameterizations of the nonlinear energy transfer in a gravity wave spectrum. Part II: Parameterizations of the nonlinear transfer for application in wave models, J. Phys. Oceanogr., 15, 11, 1378-1391.

Holloway, G. 1980. Oceanic internal waves are not weak waves, J. Phys. Oceanogr., 10, 906-914.

Janssen, P.A.E.M., G.J. Komen, and W.J.P. de Voogt. 1984. An operational coupled hybrid wave prediction model, J. Geophys. Res., 89, C3, 3635-3654.

Jensen, M.S. 2002. Breaking of waves over a steep bottom slope, Ph.D. thesis, Hydraulics \& Coastal Engineering Laboratory, Department of Civil Engineering, Aalborg University, Denmark, ISSN 0909-4296, Series paper No. 22, 162 pp.

Komen, G.J., S. Hasselmann, and K. Hasselmann. 1984. On the existence of a fully developed wind-sea spectrum, J. Phys. Oceanogr., 14, 8, 1271-1285.

Kuik, A.J., G.Ph. van Vledder, and L.H. Holthuijsen. 1988. A method for the routine analysis of pitchand-roll buoy wave data, J. Phys. Oceanogr., 18, 7, 1020-1034.

Longuet-Higgins, M.S. 1974. On the mass, momentum, energy and circulation of a solitary wave, Proc. Roy. Soc., A, 337, 1 - 13.

Madsen, P.A., and O.R. Sørensen. 1993. Bound waves and triad interactions in shallow water, Ocean Eng., 20, 4, 359-388.

McCowan, J. 1894. On the highest wave of permanent type, Phil. Mag., Series 5, 32, 351-358. Cited by Longuet-Higgins (1974).

Nelson, R.C. 1997. Height limits in top down and bottom up wave environments, Coast. Eng., 32, 247254.

Rattanapitikon, W. 2007. Calibration and modification of energy dissipation models for irregular wave breaking, Ocean Eng., 34, 1592 - 1601. 
Ris, R.C., N. Booij, and L.H. Holthuijsen. 1999. A third-generation wave model for coastal regios, Part II, Verification, J. Geophys. Res., 104, C4, $7667-7681$.

Rogers, W.E., P.A. Hwang, and D. Wang. 2003. Investigation of wave growth and decay in the SWAN model: three regional-scale applications, J. Phys. Oceanogr., 33, 366-389.

Salmon, J.E., L.H. Holthuijsen, M. Zijlema, G.Ph. van Vledder, and J.D. Pietrzak. 2014. Scaling depthinduced wave-breaking in third-generation spectral wave models. Manuscript submitted for publication.

Smith, J.M. 2004. Shallow-water spectral shapes, Proc. of 29th Int. Conf. Coastal Engng., World Scientific, 206-217.

Van der Westhuysen, A.J.. 2010. Modeling of depth-induced wave breaking under finite depth wave growth conditions, J. Geophys. Res., 115, C01008, doi: 10.1029/2009JC005433.

Van Vledder, G.Ph., J.G. de Ronde, and M.J.F. Stive. 1994. Performance of a spectral wind-wave model in shallow water, Proc. of $24^{\text {th }}$ Int. Conf. on Coastal Engng., ASCE, 761-774.

Van Vledder, G.Ph., J. Groeneweg, and A.J. van der Westhuysen. 2008. Numerical and physical aspects of wave modeling in a tidal inlet, Proc. of $31^{\text {st }}$ Int. Conf. Coastal Engng., World Scientific, 424-436.

WAMDI group, 13 authors. 1988. The WAM model - a third generation ocean wave prediction model, J. Phys. Oceanogr., 18, 12, 1775-1810.

Zijlema, M., and A.J. van der Westhuysen. 2005. On the convergence behaviour and numerical accuracy in stationary SWAN simulations of nearshore wind wave spectra, Coast. Eng., 52, 237256.

Zijlema, M., G.Ph. van Vledder, and L.H. Holthuijsen. 2012. Bottom friction and wind drag for spectral wave models, Coast. Eng., 65, 19-26. 